\title{
Provider Weight-Bias and How It Contributes to Healthcare Disparities of Obese Patients
}

\author{
Joy Dugan, MPH, PA-C*, Amanda Flohr, PA-S, Heather Hosack-Gardiner , MPH, PA-C, and Suzanne Little, MPH, PA-C \\ Touro University, USA
}

*Corresponding author: Joy Dugan, Associate Program Director, Joint MSPAS/MPH Program, California, USA

Submission: 海 October 22, 2018; Published: 非 November 13, 2018

\section{Introduction}

The prevalence of obesity has progressively increased over the past four decades for people of all ages, genders, racial and ethnic groups, and educational levels [1-4]. According to the World Health Organization (WHO), as of 2016, the worldwide obesity rate has tripled since 1975 with more than 650 million obese adults and 1.9 billion overweight adults [5]. Additionally, over 41 million children under the age of 5 and 340 million children aged 5-19 were overweight or obese in 2016 [5]. Evidence shows that children with obesity tend to remain obese into adulthood; thus, the percentage of obese adults will continue to rise in the coming decades, equating to $38 \%$ adults with obesity and and additional $20 \%$ adults being overweight globally by $2030[1,2,6]$.

Obesity has been well documented as a risk factor for a multitude of chronic diseases including cancer, coronary artery disease, cerebrovascular disease, dyslipidemia, hypertension, mental illness, obstructive sleep apnea, osteoarthritis, and type 2 diabetes [7]. In the United States, the Centers for Disease Control and Prevention (CDC) reported overweight and obesity direct and indirect healthcare costs to be over US $\$ 147$ billion dollars in 2008 $[7,8]$. Worldwide, the estimated healthcare cost associated with US $\$ 2$ trillion dollars [9].

The chronic nature of the co-morbidities suffered by people with obesity requires these patients to see their medical providers multiple times a year. They depend on their providers to manage their conditions without the burden of discrimination. This paper will review how weight bias among society and American health care providers affect patient management and treatment, potentially contributing to worse health outcomes.

\section{Background of Disparity}

Overweight or obese individuals within the United States experience a type of socially accepted discrimination, that is only increasing in prevalence, known as the weight bias [10-13]. The weight bias occurs when individuals who are overweight or obese are attributed with lesser values and traits. For example, individuals with obesity are associated with the negative societal stereotypes of lazy, weak-willed, non-reliant, undisciplined, emotionally unstable, slow thinkers, and poor role-models [1114]. Whereas non-obese patients are characterized as beautiful, emotionally stable, disciplined, motivated, and cleanly [14,15]. This stigmatization leads to poorer societal outcomes, such as being less likely to be hired for the same job, make the same salary, and even earn college acceptance than their non-obese counterparts because of their physical appearance $[14,15]$.

This trend of discrimination against obese individuals is prevalent throughout the medical world as well. Studies show that intentionally or unintentionally obesity may influence a healthcare provider's judgement, treatment, and practice [13-16]. The issue of weight bias in medical care settings and its professionals is of great concern due to the potential creation of a barrier between some of the most chronically ill individuals and those who are supposed to be caring for them [13,17-18]. Effective and patient centered care can be compromised if providers feel unwilling or unable to work with obese individuals, or if an obese individual feels discriminated against by their medical provider.

These critical attitudes are so prevalent in our society through the media, educational system, and even the medical system, that individuals begin to internalize and accept the very negative message that society is giving them [19]. This internalized negative self-image, along with actual and perceived provider weight- bias, has been shown to cause postponement in obese patients seeking medical care and completing regular medical screenings than nonobese counterparts, potentially leading to more advanced disease presentations $[12,20]$. In addition, these patients are at increased risk of mental health disorders, such as depression, stress, anxiety, and maladaptive eating habits [21-24]. If overweight and obese patients are not receiving the proper counseling and referrals, they are at an even further risk of morbidity and mortality from the course of these chronic and debilitating diseases, which through modern medicine can otherwise be managed and even prevented through proper care.

Additionally, the patients' perception of their provider has a great influence on whether he or she seeks care. One reason for this is that obese individuals are embarrassed or feel like their providers have negative perceptions about them due to their weight. Patients have reported embarrassment with getting weighed at each visit, needing larger equipment to perform general exams, and feeling disrespected because of their size [25]. Because of these feelings, 
preventive services, such as mammograms and Pap smears may be delayed or avoided in obese women compared to their non-obese counterparts $[18,26,27]$. Unfortunately, delays in these services could lead to life-threatening consequences and poorer overall health outcomes.

A study performed by Teachman and Brownell [28] found that healthcare providers have strong implicit negative attitudes and stereotypes of obese individuals. The same study indicated that providers associated bad and lazy with obesity and described normal weight individuals as good and motivated. Additionally, body mass index (BMI) and physician respect are inversely related but also that a higher BMI invokes a negative estimation of the patient by the provider [29]. Furthermore, only $56 \%$ primary care physicians felt competent in treating obesity [30]. In another study, physicians did feel capable of managing patients with obesity under-utilized tools to promote healthy changes in their patients lifestyles [31]. Those physicians felt that weight and obesity management is exasperating and unsatisfying professionally and that many believed patients were unmotivated to make behavioral changes [31]. Furthermore, tools to help promote healthy changes were underutilized with patients. These biases held by providers and other healthcare professionals could negatively affect clinical judgment, as well as, discourage obese people from seeking care $[12,14,22]$.

In medical training, providers are taught to remain objective while maintaining awareness of their own personal biases in order to provide patient-centered care. However, research indicates this is quite the opposite in managing obese and overweight patients. This bias is widespread across allied healthcare fields including physicians, nurses, physician assistants, mental health disciplines, and physiotherapists, and dieticians [13,14,17,32]. In one study, nurses thought that obese persons were unsuccessful, overindulgent, lazy and experience unresolved anger [14]. The same study revealed that some nurses feel uncomfortable caring for obese patients. Another study found that medical doctors have a weight-bias that is as pervasive as the general public, which can lead to both verbal and non-verbal barriers in patient care [13]. Furthermore, this bias is prevalent among physicians that are overweight or obese, possibly indicating another danger of internalized stigmas.

Evidence from the literature suggests that a provider's weight, diet, and exercise habits influence the type of care rendered. Physicians who exercise more and maintain a healthy diet are more likely to discuss exercise, weight management, and nutrition with all their patients $[30,33]$. Furthermore, physicians who ate less fat in their diets were more likely to counsel and screen patients for high cholesterol [33]. Thus, a disparity may exist amongst obese providers in discussing exercise, nutrition, weight management, and cholesterol screening with their patients.

\section{Discussion}

Weight-based biases seem to develop as early as 3 yearsold and pursue well into adulthood [34-36]. Unfortunately, these negative attitudes seem to go unchecked during medical training as evidenced by health care professional and student biases $[17,32]$. Postgraduate students in healthcare fields, such as physician assistant, psychiatry, and clinical psychology, perceived obese individuals as lacking motivation, being non-compliant, and difficult to manage [32]. In addition, despite the awareness of weight discrimination, participants reported peers, instructors, and health providers making derogatory comments about obese individuals, furthering the idea of social acceptance of weightbiases [32]. Negative attitudes towards obese individuals has also been documented in medical students, dietitians, physicians, and nurses [18]. Poon and Tarrant [37] found that obese patients are often the target of derogatory and cynical humor by medical students and residents. A lack of sensitivity training in addition to preconceived notions about obesity originating in society's bias contribute to medical students' attitudes toward obese patients.

The presence of bias toward obese individuals may be based on superficial characteristics, but there are greater implications pertaining to the healthcare provided to patients with obesity. Several studies discovered that providers tend to spend $28 \%$ less time with patients with obesity due to feelings of the visit being wasteful, thus offering less patient education and patient-centered care [12,38,39]. Budd et al. [25] also found that physicians are more likely to order more tests for patients who are overweight despite spending less time with patients with obesity. This may lead higher medical costs due to unnecessary tests being ordered and a lack of one-on-one interviewing and counseling that may have a greater impact.

Table 1: Overweight and obesity ICD-10 Codes for providers to be used with counseling codes.

\begin{tabular}{|c|c|}
\hline Category & ICD-10 Code(s) \\
\hline Overweight & E66.3 \\
BMI & Z68.25-Z68.29 \\
\hline Obesity & E66.0 \\
Due to excess & E66.1 \\
calories & \\
Drug-induced & E66.2 \\
Morbid obesity with alveolar & E66.8 \\
hypoventilation & \\
Other & $\mathrm{E} 66.9$ \\
Unspecified & $\mathrm{Z} 68.30-\mathrm{Z} 68.45$ \\
BMI & $\mathrm{Z} 71.3$ \\
\hline Counseling & \\
services & $\mathrm{Z} 71.82$ \\
Dietary counseling and surveillance & \\
\hline Exercise counseling & \\
\hline
\end{tabular}

A standard primary care office visit in the U.S. is often only fifteen minutes, during which the provider is expected to make a take a history, perform a physical exam, make an appropriate 
treatment plan, and document this in the patient's chart [40]. The current healthcare model leaves little time to provide weight management and reduction strategies. Even if providers do perform patient education regarding weight management, exercise and diet, this time spent is often non-billable because there are multiple stipulations for reimbursement [41,42]. Table 1 includes International Statistical Classification of Diseases and Related Problems (ICD-10) codes that may be used for reimbursement of obesity and related conditions (Figure 1). There is also a lack of education in medical training on diet and exercise [43].

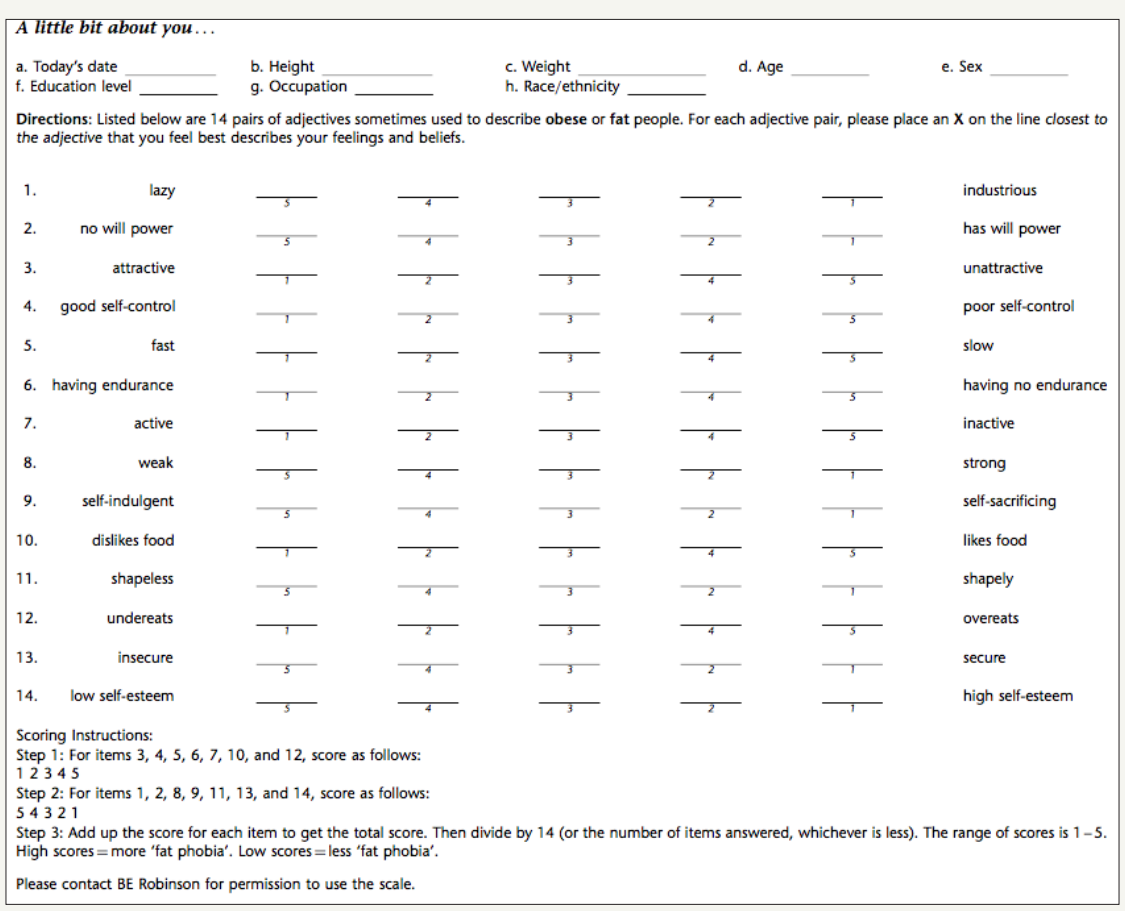

Figure 1: Fat Phobia Scale Short Form adapted from BE Robinson.

Despite the fact that many providers agree there is a need to treat obesity, most primary care physicians do not even diagnose patients with obesity or develop a weight loss plan for them $[30,44]$. In fact, it has been reported that only forty-two percent of overweight or obese patients state their provider discussed weight loss with them [45]. The same study found that patients who are advised to lose weight are significantly more likely to attempt weight loss compared to those who do not get this advice. However, many providers neglect weight loss counseling, nutrition, and management $[30,44]$. Possible hurdles on the provider's aspect may include many feelings incapable of providing the proper treatment plans and sentiments that obese patients are incompetent or noncompliant [30,32]. These barriers stemming from weight bias need to be addressed so the disparity can be minimized, and costly comorbidities recognized and treated.

On the patients' perspective barriers to seeking care and the decision to forego screening can be from the preconception and internalization that obesity is "bad." Obese patients may be reluctant to seek health care because of weight bias, which prevents early detection leading to increased healthcare costs [18]. Other studies had similar findings pertaining to overweight and obese women not receiving medical treatment or necessary cancer screenings due to negative body image and/or providers being reluctant to perform exams on obese women $[12,46]$. Reasons obese patients did not seek healthcare included: embarrassment from weigh-ins before the visit, feeling disrespected by the provider, too-small clinical equipment like gowns and blood pressure cuffs, unrequested weight loss advice, and judgmental treatment plans [25].

The gap between needing and receiving preventive care can have costly effects on a person's health, especially if that person happens to be overweight. Preventative services are in place to decrease the risk of developing life-threatening diseases such as cancer and heart disease. Delaying care can also contribute to developing co-morbidities that are strongly associated with obesity such as hypertension, hyperlipidemia, diabetes, osteoarthritis, and coronary artery disease. A person's unwillingness to seek care based on their being obese can lead to poor management of comorbidities that can lead to myocardial infarction, stroke, heart failure, extremity amputation, kidney failure, and blindness. The stigma attached to obesity needs to be reassessed by healthcare providers for obese patients to feel more comfortable during provider encounters as to prevent further morbidity.

Encouraging obese patients to have regular visits with a provider warrants increased interaction, improved trust between both parties, and increased time spent with patients as to address behavior changes that may influence a patient to lose weight. This concept has been shown in several studies that found that when providers spend more time with patients and are empathetic and encouraging in patient-directed goals rather than having a 
judgmental tone with unrealistic goals, patients are more perceptive with their provider and, therefore, more likely to have over a $10 \%$ weight loss $[18,47,48]$.

\section{Recommendation}

Since weight-bias exists amongst providers giving care to obese patients, the provider's perception must be improved. This should occur early in health professional training programs since many of these students, with youth and inexperience, already hold a weightbias [18]. These negative biases regarding obesity can potentially cause adverse effects on delivery of care and must be addressed.

Utilizing the well-validated Fat Phobia Scale Short Form (Figure 1) at the beginning of medical training can help students determine their bias towards obese patients $[19,49]$. If students are allowed to self-score the Fat Phobia Scale, it would be a step towards reducing prejudice and stereotyping since one must first recognize their own prejudices in order to combat them [49]. The scale contains 14 pairs of adjectives to describe obese people. A numerical value of 1-5, where 1 is the least biased and 5 is most biased, is applied to each answer to the 14 questions. An average score is calculated from the total responses. Based on previous validation studies, a score of 4.4 indicates a high level of negative attitudes or stereotypes towards obese individuals. A score of 3.6 is average, while 2.5 or less is positive or neutral attitude regarding obese individuals.

Another method is using Project Implicant's Weight Implicit Association Test. This test has two parts: one in which the user associate's images of overweight individuals with negative word categories and images of thin people with positive word categories; this is then reversed in the second part. The test is scored by measuring the difference between the average response time for each part. This validated test provides distinguishable information from self-reports $[13,50,51]$.

Interventions can also be extended beyond those in medical training and to practicing clinicians. One suggestion is to make the clinic environment more welcoming to those with obesity. For example, having larger-sized blood pressure cuffs and pelvic examination equipment more readily available, larger chairs in the waiting room, and optional weigh-ins are possible steps that can be taken to improve the comfort of an encounter [12,52]. During the encounter itself, the provider can take a less judgmental stance by empathizing with the patient and focusing less on their actual weight and more on healthy behaviors, such as increased fruit and vegetable intake, remaining physically active, improving sleep behavior, and reducing stress [52]. This can improve the providerpatient relationship by fostering understanding and openness leading to behavior changes [12].

Behavioral intervention for obesity should be consistent with the "5-As", as recommended by the United States Preventive Services Task Force [53]. The "5-As" include Assess the health risks, Advise on behavior change, Agree on treatment goals and willingness to change, Assist in the patient achieving the goals with medical treatments and behavior change techniques, Arrange follow-up contacts for ongoing support and utilize specialist referrals as needed.

\section{Conclusion}

Obesity is an international epidemic. With nearly one third of the American population falling into the category of a BMI greater than thirty and a multitude of health consequences strongly correlated to obesity, it is of the utmost importance to ensure prevention and proper treatment for overweight and obese individuals. It is necessary to develop a medical work-force who is compassionate, caring, non-judgmental, and unbiased toward this ever-increasing population. Obese individuals often require significantly more office visits, polypharmacy, and increased referrals to specialists than non-obese individuals because of their increased risk for comorbidities. Considering the increased healthcare need in this population, it is imperative for providers to offer non-judgmental weight loss counseling, including nutritional information and exercise counseling, and specialist referrals to help empower this population, to improve their overall health, as well as decrease their risk of subsequent disease processes.

Changing negative views and stereotypes of the obese population amongst providers may prove to be a difficult challenge as these stereotypes are deeply ingrained in our culture as well as in medicine. Difficulties in properly addressing obesity in a sensitive and non-judgmental manner in a time-constrained office visit can prove frustrating for the provider and for the patient. By making essential changes in the way in which medicine is taught and changes in the way in which providers receive continuing medical education aiming to creating more sensitive and unbiased providers through tools such as role playing and use of the Fat Phobia Scale, and by creating tools and resources to properly address this population, healthcare providers would be more inclined to treat this population and to make positive changes in the overall health of these individuals' lives.

\section{References}

1. Hruby A, Hu FB (2015) The epidemiology of obesity: A big picture. Pharmacoeconomics 33(7): 673-689.

2. Mokdad AH, Ford ES, Bowman BA, Dietz WH, Vinicor F, et al. (2003) Prevalence of obesity, diabetes, and obesity-related health risk factors. JAMA 289(1): 76-79.

3. Ogden CL, Carroll MD, Fryar CD, Flegal KM (2015) Prevalence of obesity among adults and youth: United States, 2011-2014. NCHS data brief 219: 1-8.

4. Eidell JC, Halberstadt J (2015) The occurrence of obesity in an individual or in populations is a result of combinations of factors at multiple levels of influence. Ann Nutri Metab 66(2): 7-12.

5. World Health Organization. Obesity and overweight. World Health Organization.

6. Sahoo K, Sahoo B, Choudhury AK, Sofi NY, Kumar R, et al. (2015) Childhood obesity: causes and consequences. J Family Med Prim Care 4(2): 187-192.

7. Adult obesity causes \& consequences. Centers for Disease Control and Prevention.

8. Finkelstein EA, Trogdon JG, Cohen JW, Dietz W (2009) Annual medical spending attributable to obesity: Payer-and service-specific estimates. Health Aff 28(5): w822-s831.

9. Tremmel M, Gertham U, Nilsson PM, Saha S (2017) Economic burden of obesity: A systematic literature review. Int J Environ Res 14(4): 435. 
10. Alberga AS, Mayhew RS, Ranson KM, McLaren L (2016) Weight bias: A call to action. J Eat Disord 4(34): 1-6.

11. Carels RA, Eizenman DR (2010) Individual differences and weight bias: Do people with an anti-fat bias have a pro-thin bias? Body Image $7(2)$ : 143-148.

12. Phelan SM, Burgess DJ, Yeasel MW, Hellerstedt WL, Griffin JM, et al (2015) Impact of weight bias and stigma on quality of care and outcomes for patients with obesity. Obes Rev 16(4): 319-326.

13. Sabin JA, Marini M, Nosek BA (2012) Implicit and explicit anti-fat bias among a large sample of medical doctors by BMI, race/ethnicity and gender. PLoS ONE 7(11): e48448.

14. Puhl R, Brownell KD (2001) Bias, discrimination, and obesity. Obesity 9(12): 788-805.

15. Brien KS, Latner JD, Ebneter D, Hunter JA (2012) Obesity discrimination: The role of physical appearance, personal ideology, and anti-fat prejudice. Int J Obes (Lond) 37(3): 455-460.

16. Brownell KD, Pulh R (2003) Stigma and discrimination in weight management and obesity. The Permanente Journal 7(3): 21-23.

17. Hales C, Gray L, Russell L, MacDonald C (2018) A qualitative study to explore the impact of simulating extreme obesity on health care professionals' attitudes and perceptions. Ostomy Wound Manage 64(1): 18-24.

18. Schwartz MB, Chambliss H, Brownell KD, Blair SN, Billington C (2003) Weight bias among health professionals specializing in obesity. Obes Res 11(9): 1033-1039.

19. Bacon JG, Scheltema KE, Robinson BE (2001) Fat phobia scale revisited: The short form. Int J Obes 25(2): 252-257.

20. Rand CW, MacGregor AM (1990) Morbidly obese patients' perceptions of social discrimination before and after surgery for obesity. South Med J 83(12): 1390-1395.

21. Himmelstein MS, Puhl RM, Quinn DM (2018) Weight stigma in men: What, when, and by whom? Obesity 26(6): 968-976.

22. Puhl RM, Brownell KD (2006) Confronting and coping with weight stigma: An investigation of overweight and obese adults. Obesity (Silver Spring) 14(10):1802-1815

23. Tomiyama AJ (2014) Weight stigma is stressful. A review of evidence for the cyclic obesity/weight-based stigma model. Appetite 82: 8-15.

24. Wu YK, Berry DC (2017) Impact of weight stigma on physiological and psychological health outcomes for overweight and obese adults: A systematic review. J Adv Nurs 74(5): 1030-1042.

25. Budd GM, Mariotti M, Graff D, Falkenstein K (2011) Health care professionals' attitudes about obesity: An integrative review. App Nurs Res 25(3): 127-137.

26. Wee CC, McCarthy EP, Davis RB, Philips RS (2000) Screening for cervica and breast cancer. Is obesity an unrecognized barrier to preventative care? Ann Intern Med 132(9): 697-704.

27. Olson CL, Schumaker HD, Yawn BP (1994) Overweight women delay medical care. Arch Fam Med 3(10): 888-892.

28. Teachman BA, Brownell KD (2001) Implicit anti-fat bias among health professionals: is anyone immune? Int J Obes Relat Metab Disord 25: $1525-1531$.

29. Gudzune KA, Huizinga MM, Beach MC, Cooper LA (2012) Obese patients overestimate physicians' attitudes of respect. Patient Educ Couns 88(1): 23-28.

30. Jay M, Kalet A, Tavinder A, Michelle Mc, Mary J, et al. (2009) Physicians' attitudes about obesity and their associations with competency and specialty: A cross-sectional study. BMC Health serv Res 9: 106-116.
31. Campbell K, Engel H, Timperio A, Cooper C, Crawford D (2000) Obesity management: Australian general practitioners' attitudes and practices. Obes Res 8(6): 459-466.

32. Puhl RM, Luedicke J, Grilo CM (2014) Obesity bias in training: Attitudes, beliefs, and observations among advanced trainees in professional health disciplines. Obesity (Silver Spring) 22(4): 1008-1015.

33. Rothenberg FE, Lewis C, Belodoff BF (2000) Correlates of physicians' prevention-related practices. Findings from the Women Physicians' Health Study. Arch Fam Med 9(4): 359-367.

34. Skinner AC, Payne K, Perrin AJ, Panter AT, Howard JB, et al. (2017) Implicit weight bias in children 9 to 11 years. Pediatrics 140(1): 1-6.

35. Harrison S, Rowlinson M, Hill AJ (2016) No fat friend of mine: Young children's responses to overweight and disability. Body Image 18: 65-73.

36. Worobey J, Worobey HS (2014) Body-size stigmatization by preschool girls: In a doll's world, it is good to be Barbie. Body Image 11(2): 171174.

37. Poon MY, Tarrant M (2009) Obesity: Attitudes of undergraduate student nurses and registered nurses. J Clin Nurs 18(16): 2355-2365.

38. Hebl MR, Mason MF (2003) Weighing the care: Patients' perceptions of physician care as a function of gender and weight. Int J Obese Relat Metab Disord 27(2): 269-275.

39. Hebl MR, Xu J (2001) Weight the care: physicians' reactions to the size of a patient. Int J Obes Relat Metab Disord 25(8): 1246-1252.

40. Linzer M, Bitton A, Tu S, Plews OM, Horowitz KR, et al. (2015) The end of the 15-20-minute primary care visit. J Gen Intern Med 30(11): 15841586 .

41. U.S. Centers for Medicare \& Medicaid Services. Nutrition therapy services (medical). Medicare.gov: The Official U.S. Government Site for Medicare website.

42. Reimbursement policy (2018) Weight management care, nutritional counseling and medical nutrition therapy. Blue Cross Blue Shield of Minnesota.

43. Adams KM, Kohlmeier M, Zeisel SH (2010) Nutrition education in us medical schools: Latest update of a national survey. Acad Med 85(9): 1537-1542.

44. Bardia A, Holtan SG, Slezak JM, Thompson WG (2007) Diagnosis of obesity by primary care physicians and impact on obesity management. Mayo Clin Proc 82(8): 927-932

45. Galuska DA, Will JC, Serdula MK, Ford ES (1991) Are healthcare professionals advising obese patients to lose weight? JAMA 282(16): 1576-1578.

46. Adams CH, Smith NJ, Wilbur DC, Grady KE (1993) The relationship of obesity to the frequency of pelvic examinations: do physician and patient attitudes make a difference? Women's Health J 20(2): 45-57.

47. Blanton C, Brooks JK, McKnight L (2015) Weight bias in university health professions students. J Allied Health 45(3): 212-18.

48. Gudzune KA, Bennett WL, Cooper LA, Bleich SN (2014) Perceived judgment about weight can negatively influence weight loss: A crosssectional study of overweight and obese patients. Prev Med 62: 103-107.

49. Wolf C (2010) Physician Assistant students' attitudes about obesity and obese individuals. J Physician Assist Educ 21(4): 37-40.

50. Cunningham WA, Preacher KJ, Banaji MH (2001) Implicit attitude measures: Consistency, stability, and convergent validity. Psychol Sci 12(2): 163-170.

51. Elgoff B, Schwerdtfeger A, Schmukle SC (2005) Temporal stability of the implicit association test- anxiety. J Pers Assess 84(1): 82-88. 
52. Tomiyama AJ, Carr D, Granberg EM, Major B, Robinson E, et al. (2018) How and why weight stigma drives the obesity epidemic and harms health. BMC Med 16(123): 1-6.

53. The five As. AACE Obesity Resource Center.

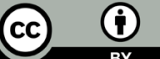

Creative Commons Attribution 4.0 International License

For possible submissions Click Here

Submit Article

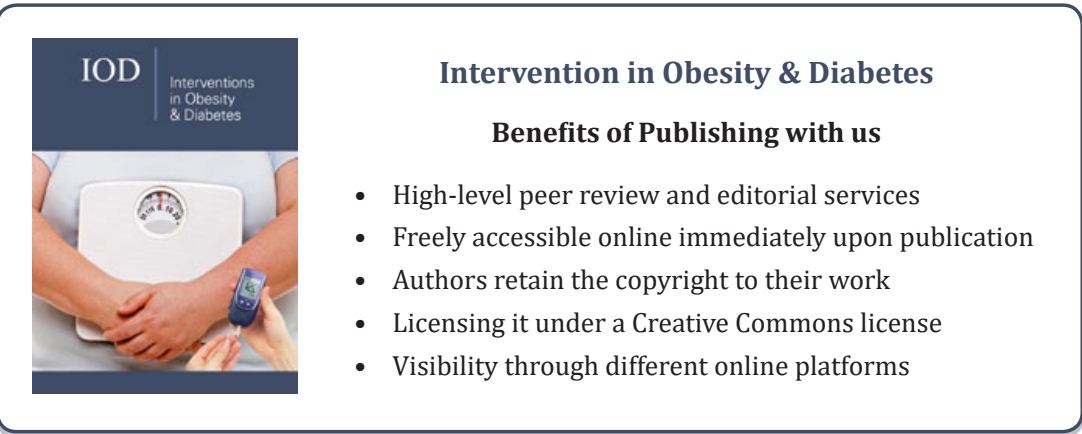

